(C) 1982. The Genetical Society of Great Britain

\title{
ADDITIVE POLYGENIC FORMULATION OF HAMILTON'S MODEL OF KIN SELECTION*
}

\author{
KENICHI AOKI \\ National Institute of Genetics, Mishima, Shizuoka-ken 411, Japan
}

Received 16.iii.82

\section{SUMMARY}

Hamilton's condition for the evolution of altruism by kin selection is rederived on an additive polygenic model. The approach uses intraclass correlation. It is then shown that the mean inclusive fitness is non-decreasing. The differences between and the relative efficacy of kin and group selection are discussed.

\section{INTRODUCTION}

HAMiLton (1964) generalized Fisher's (1930) and Haldane's (1955) seminal argument to show heuristically that altruism between same-generation kin would evolve if the following approximate inequality were satisfied:

$$
b r>c .
$$

Here, $r$ is the correlation between the additive genotypic values of the interacting kin as evaluated from the pedigree. In Hamilton's model of benefit dispensation, an altruist suffers a cost in fitness $c$ while donating a total benefit $b$, which is distributed evenly among the recipients. A recipient experiences its share of the benefit as an increment to its fitness; the total benefit to a recipient is the sum of increments received from all sources. Inequality (1) was first proposed to hold in a random mating population with discrete non-overlapping generations (Hamilton, 1964), and then extended to accommodate inbreeding (Hamilton, 1971, 1972). For symmetrical relationships, which we alone consider in this paper, $r$ is equal to $2 f_{1} /\left(1+f_{0}\right)$, where $f_{0}$ is the inbreeding coefficient, and $f_{1}$ is the coefficient of consanguinity of the interacting kin (Wright, 1922). Based on an one-locus two-allele genetic model, Michod (1979) and Aoki (1981) have shown that (1) is valid under arbitrary dominance when mating is random, whereas it holds only for additively acting alleles when there is inbreeding.

Although it is widely believed that most behavioural traits are multifactorial, most analyses of the evolution of altruism have been monogenic. Notable exceptions are the kin selection model of Yokoyama and Felsenstein (1978) and the group selection models of Crow and Aoki (1982) and Aoki (1982). It is the purpose of this note to show that Hamilton's inequality (1) can be straightforwardly derived on an additive polygenic model. The finiteness of the number of interacting kin is explicitly considered and shown not to affect the result. The formulation is mathematically quite similar to the group selection model of Crow and Aoki (1982). However,

\footnotetext{
* This is contribution number 1429 from the National Institute of Genetics, Mishima, Shizuoka-ken 411, Japan.
} 
there are important differences to be mentioned below, which confirm the difference between kin and group selection. With this formulation, it is also possible to provide a simple proof of the proposition that the mean inclusive fitness is non-decreasing (Hamilton, 1964).

\section{Derivation}

Let $C$ be the value of a quantitative behavioural trait in diploid organisms, such as the propensity to perform altruism or the intensity of altruism performed. An individual with character value $C$ is assumed to suffer a cost in fitness $c C$ and to dispense benefit $b C$ according to Hamilton's model of benefit dispensation. We view the altruistic interactions as affecting survival or fertility. The trait is assumed to be determined by a number of genes and by the environment. The genes are assumed to act additively within and between loci, and the environment is assumed not to interact or be correlated with the genotype.

Altruistic interactions are assumed to occur within temporary associations of kin which are formed after birth and prior to reproduction, and which, for want of a better term, we will henceforth call kin groups. (A kin group corresponds to "trait group" of Wilson (1975).) An important example of a kin group is a group of siblings. Within an arbitrary kin group $s$, let the mean and the genetic variance of $C$ be $\bar{C}_{s}$ and $V_{s}$, respectively. We assume that the environmental component has zero mean deviation within each kin group. Then the environment can be safely ignored in all subsequent arguments; we will use $C$ interchangeably to mean the genotypic value or the phenotypic value depending on the context. Let $P_{s}$ be the proportion of all individuals that belong to kin group $s$. We assume a large number of kin groups.

The change in the population mean $\bar{C}$ of the trait value under selection is given by,

$$
\begin{aligned}
\Delta \bar{C} & =\sum_{s} P_{s}^{\prime} \bar{C}_{s}^{\prime}-\sum_{s} P_{s} \bar{C}_{s} \\
& =\sum_{s} P_{s}^{\prime} \cdot \Delta \bar{C}_{s}+\sum_{s} \bar{C}_{s} \cdot \Delta P_{s}
\end{aligned}
$$

where we use the discrete time formulation and a prime indicates the values after selection. When selection is on viability differences, the meaning of (2) is straightforward. When selection is on fertility differences, $P_{s}^{\prime}$ and $\bar{C}_{s}^{\prime}$ can be interpreted as the proportion and twice the mean value of gametes contributed by kin group $s$ to the next generation. In either case, the additivity assumption on gene action ensures that there is no change in $\bar{C}$ due to the reshuffling of genes at reproduction. Thus (2) gives the change per generation in $\bar{C}$.

When altruistic interactions involve only members of one sex, the magnitude of $\Delta \bar{C}$ is reduced by as much as $\frac{1}{2}$ depending on the level of inbreeding. Its sign, however, remains unaffected.

The first term on the right hand side of (2) represents the change in $\bar{C}$ due to within kin group selection, while the second term represents the effect of between kin group selection. Such an approach in which the effect 
of selection is divided into a within group component and a between group component has been previously used by Price $(1970,1972)$, Hamilton (1975), Wade (1980), Crow and Aoki (1982), and Aoki (1982).

For any kin group $s$, we can modify the continuous time derivation of Crow and Nagylaki (1976) (see also Robertson, 1966) to obtain

$$
\Delta \bar{C}_{s}=2 \sum_{k} \sum_{i} p_{i s}^{(k)} \gamma_{i s}^{(k)} w_{i s}^{(k)} / \bar{W}_{s}
$$

where $p_{i s}^{(k)}$ is the frequency, $\gamma_{i s}^{(k)}$ is the average effect on trait value, and $w_{i s}^{(k)}$ is the average excess in fitness of the $i$ th allele at the $k$ th locus contributing to the trait; and $\bar{W}_{s}$ is the mean Wrightian fitness of kin group $s$. The numerator of the term on the right hand side can be interpreted as a covariance, although this interpretation will not prove to be useful in this paper.

To obtain an explicit form for (3), we assume for notational brevity that the trait is determined by two loci, but the result is readily generalizable to an arbitrary number of loci. Let $A_{i}^{(k)}$ stand for the $i$ th allele at the $k$ th locus. The genotypic trait value of genotype $A_{i}^{(1)} A_{j}^{(1)} A_{k}^{(2)} A_{l}^{(2)}$, whose ordered frequency (Nagylaki, 1977) we denote by $P_{i j k l, s}$, can be decomposed as

$$
C_{i j k l}=\bar{C}_{s}+\gamma_{i s}^{(1)}+\gamma_{j s}^{(1)}+\gamma_{k s}^{(2)}+\gamma_{l s}^{(2)} .
$$

The fitness of this genotype, as measured from an arbitrary standard $W_{0}$ is

$$
W_{i j k l, s}=W_{0}+b \bar{C}_{s}-c C_{i j k l} .
$$

It is assumed for now that kin group size is large enough that factors involving its reciprocal can be ignored.

After some straightforward manipulation, it can be shown that the mean fitness of kin group $s$ is

$$
\bar{W}_{s}=W_{0}+(b-c) \bar{C}_{s}
$$

and finally

$$
2 \sum_{k} \sum_{i} p_{i s}^{(k)} \gamma_{i s}^{(k)} w_{i s}^{(k)}=-c \sum_{i} \sum_{j} \sum_{k} \sum_{l} P_{i j k l, s}\left\{\gamma_{i s}^{(1)}+\gamma_{j s}^{(1)}+\gamma_{k s}^{(2)}+\gamma_{l s}^{(2)}\right\}^{2} .
$$

The sum on the right-hand side of (7) is the genetic variance of kin group s. Thus

$$
\Delta \bar{C}_{s}=-c V_{s} / \bar{W}_{s}
$$

From elementary considerations, it can be seen that

$$
P_{s}^{\prime}=P_{s} \bar{W}_{s} / \bar{W}
$$

where $\bar{W}=\sum_{s} P_{s} \bar{W}_{s}$

Substituting (6), (8), and (9) into (2), we obtain

$$
\bar{W} \cdot \Delta \bar{C}=-c \sum_{s} P_{s} V_{s}+(b-c) \sum_{s} P_{s}\left(\bar{C}_{s}-\bar{C}\right)^{2}
$$


which can be conveniently written as

$$
\bar{W} \cdot \Delta \bar{C}=-c V_{w}+(b-c) V_{b} .
$$

Here, $V_{w}$ and $V_{b}$ are by definition the within kin group and between kin group genetic variances of the trait value. The total genetic variance of the population is $V_{t}=V_{w}+V_{b}$.

The intraclass correlation between genotypic trait values in a kin group is

$$
t=V_{b} /\left(V_{w}+V_{b}\right)
$$

On rearranging (11) and substituting (12) therein, we have

$$
\bar{W} \cdot \Delta \bar{C}=V_{t}(b t-c) \text {. }
$$

Thus the condition for the mean trait value $\bar{C}$ to increase is

$$
t>c / b \text {. }
$$

We note that (13) and hence (14) hold exactly regardless of any assumptions on linkage, linkage disequilibrium, or inbreeding, and under the action of selection.

If we take the finiteness of kin group size into explicit consideration and assume that each kin group has the same size $N$ at conception, then (5) should be replaced by

$$
W_{i j k l, s}=W_{0}+\frac{b N}{N-1} \bar{C}_{s}-\left(\frac{b}{N-1}+c\right) C_{i j k l}
$$

since according to Hamilton's model an altruist does not dispense benefit to itself. The condition for $\bar{C}$ to increase becomes

$$
\frac{N}{N-1}\left(t-\frac{1}{N}\right)>c / b .
$$

Recall that $t$ has been defined as an intraclass correlation. It is straightforward to show that the left hand side of (16) is the corresponding product moment correlation between two randomly chosen individuals both from the same kin group. The two types of correlation need not be distinguished if kin group size is infinite. However, as (16) indicates, the relevant correlation, when as in this case the distinction is necessary, is the product moment correlation, in terms of which (14) and (16) are identical. Contrary to what has sometimes been claimed, finiteness of kin group size does not render the evolution of altruism more difficult. The above can readily be generalised to the case of differing kin group size.

Henceforth we assume that the loci determining the trait are loosely linked, so as to avoid any complications of linkage disequilibrium which may arise by random drift or selection. To be explicit, we assume that the population as a whole is in linkage equilibrium, although each kin group need not (and usually will not) be. Recall that the correlation in inequality (14) (and also (16)) involves selection and therefore differs from correlation computed according to pedigree. However, if we assume, as we do, that many loci contribute to the trait and that linkage equilibrium holds between these loci, then the selection acting on each locus should be weak and gene frequency change slow. Therefore the discrepancy should be small. Thus we have proved the approximate validity of (1) on an additive polygenic 
model. We note that the biologically interesting interpretation of $r$ as the fraction of genes identical by descent that are shared by the interacting kin also requires the assumption of linkage equilibrium (see $e . g$., Crow and Kimura 1970, pp. 136-138).

We now show that the mean inclusive fitness of the population is non-decreasing. The proposition is valid to the same order of approximation as (1). An individual with trait value $C$ has by definition (Hamilton, 1964) the inclusive fitness

$$
R=W_{0}+b C r-c C
$$

regardless of the kin group to which it belongs. Thus the change in the mean inclusive fitness $\bar{R}$ per generation is

$$
\Delta \bar{R}=(b r-c) \Delta \bar{C} .
$$

Recall that $\Delta \bar{C}>0$ if $r>c / b$ and $\Delta \bar{C}<0$ if $r<c / b$. In either case, we have $\Delta \bar{R}>0$. Thus including the case of $r=c / b$, we have

$$
\Delta \bar{R} \geqq 0 \text {. }
$$

Note by comparison that the mean fitness of the population is not necessarily non-decreasing since

$$
\Delta \bar{W}=(b-c) \Delta \bar{C}
$$

\section{Discussion}

The crucial step in the intraclass correlation approach to the proof of (1) is equation (7). This step was possible because of the special form of benefit dispensation assumed (Hamilton, 1964), in which the fitness is a linear function of the genotypic trait value (see equation (5)). Nevertheless, the algebraic machinery developed here can be successfully applied to more complicated models of benefit dispensation. In general, however, it is necessary to assume both approximate Hardy-Weinberg proportions and linkage equilibrium within each group in order to obtain a useful counterpart to (7) (Crow and Aoki, 1982). Such assumptions are realistic if the group is a partially isolated random mating unit according to the usual population genetical connotation of a group (Wright, 1980 and earlier; Maynard Smith, 1976., "traditional group" of Wade 1978), but are rarely applicable to a temporary association of kin. With the assumption of Hardy-Weinberg proportions,

$$
2 \sum_{k} \sum_{i} p_{i s}^{(k)} \gamma_{i s}^{(k)} w_{i s}^{(k)}
$$

is proportional to the additive genetic variance. With the assumption of linkage equilibrium, the additive genetic variance is equivalent to the genetic variance $V_{s}$.

These considerations indicate that there are differences between a "group" and a "kin group", from the standpoint of population genetics as well as common sense biology, and that it is useful to keep the distinction clear. However, at least for the Hamilton model of benefit dispensation, the arguments leading up to (14) are equally applicable to group selection as to kin selection. In group selection, the genetic correlation is $2 f_{0} /\left(1+f_{0}\right)$, 
or if equilibrium is achieved between island model type migration and random drift roughly $1 /\left(1+2 M_{e}\right)$ where $M_{e}$ is the effective number of migrants per generation (Crow and Aoki, 1982; see also Hamilton, 1975).

Hamilton $(1964,1971,1972)$ fully realized that (1) was an approximation, valid when gene frequency change per generation is slow. Since he based his arguments on a one-locus model, he had to assume weak selection. Our polygenic formulation has the advantage that even when overall selection is strong, per locus selection will be weak. In this respect, a polygenic model has a double advantage over a monogenic model in that it is at once genetically more realistic and permits a wider range of selection intensities.

The practically most important example of a temporary kin group is a sibling group. In a random mating population $r=\frac{1}{2}$. Another often encountered example is a half-sib group sharing a common mother. Let each half-sib group consist of $h$ full-sib subgroups of equal size $n$. Then the genetic correlation in the half-sib group is

$$
r=\frac{n-1}{h n-1} \cdot \frac{1}{2}+\frac{(h-1) n}{h n-1} \cdot \frac{1}{4}
$$

We note that these two values of $r$ will usually be much higher than $1 /\left(1+2 M_{e}\right)$ unless $M_{e}$ is small. This is possibly true even if we correct for the fact that migration usually occurs between adjacent colonies (Crow and Aoki, 1982). Thus unless there are compensatory differences in $b$ and $c$, kin selection is probably a more potent force than group selection.

\section{REFERENCES}

AOKI, K. 1981. Algebra of inclusive fitness. Evolution, 35, 659-663.

AOKI, K. 1982. Polygenic altruism and extinction group selection. Jpn. J. Genet., 57, 297-300 CROW, J. F., AND AOKI, K. 1982. Group selection for a polygenic behavioral trait: a differential proliferation model. Proc. Natl. Acad. Sci. USA, 79, 2628-2631.

CROW, J. F., AND KIMURA, M. 1970. An Introduction to Population Genetics Theory. Harper and Row, New York.

CROW, J. F., AND NAGYLAKI, T. 1976. The rate of change of a character correlated with fitness. Amer. Natur., 110, 207-213.

FISHER, R. A. 1930. The Genetical Theory of Natural Selection. Clarendon Press, Oxford.

HALDANE, J. B. S. 1955. Population genetics. New Biology, 18, 34-51.

HAMILTON, W. D. 1964. The genetical evolution of social behavior. J. Theor. Biol., 7, 1-52.

HAMILTON, W. D. 1971. Selection of selfish and altruistic behavior in some extreme models. In Eisenberg, J. F., and Dillon, W. S. (eds.) Man and Beast : Comparative Social Behavior, Smithsonian Inst. Press, Washington, pp. 59-91.

HAMILTON, W. D. 1972. Altruism and related phenomena, mainly in social insects. Ann. Rev. Ecol. Syst. 3, 193-232.

HAMILTON, W. D. 1975. Innate social aptitudes in man: an approach from evolutionary genetics. In Fox, R. (ed.) Biosocial Anthropology, Wiley, New York, pp. 133-155.

MAYNARD SMITH, J. 1976. Group selection. Quart. Rev. Biol., 51, 277-283.

MICHOD, R. 1979. Genetical aspects of kin selection: effects of inbreeding. J. Theor. Biol. $81,223-233$.

NAGYLAKI, T. 1977. Selection in One- and Two-locus Systems. Springer-Verlag, Berlin, Heidelberg, New York.

PRICE, G. R. 1970. Selection and covariance. Nature, 277, 520-521.

PRICE, G. R. 1972. Extension of covariance selection mathematics. Ann. Hum. Genet. 35, 485-490.

ROBERTSON, A. 1966. A mathematical model of the culling process in dairy cattle. Anim. Production, 8, 95-108. 
WADE, M. J. 1978. A critical review of the models of group selection. Quart. Rev. Biol., 53, $103-114$.

WADE, M. J. 1980. Kin selection: its components. Science, 210, 665-667.

WILSON, D. S. 1975. A theory of group selection. Proc. Natl. Acad. Sci. USA, 72, 143-146. WRIGHT, s. 1922. Coefficients of inbreeding and relationship. Amer. Natur. 56, 330-338.

WRIGHT, S. 1980. Genic and organismic selection. Evolution, 34, 825-843.

YOKOYAMA, S., AND FELSENSTEIN, J. 1978. A model of kin selection for an altruistic trait considered as a quantitative character. Proc. Natl. Acad. Sci. USA, 75, 420-422. 